\title{
Reconnect: Cultural Content and Audiences in the Digital Environment
}

\author{
Petya KOLEVA ${ }^{1}$, Milena BERBENKOVA ${ }^{2}$ \\ 1 Intercultura Consult, 1-A-6 Edison, 1111 Sofia, BG; (1D) petyakoleva@inter-cultura.eu \\ 2 Intercultura Consult, 1- A - 6 Edison, 1111, Sofia, BG; culture@inter-cultura.eu
}

\begin{abstract}
Several studies have addressed the challenges faced by cultural and creative professionals and businesses since the emergence of the global COVID-19 pandemic. Some success stories have surfaced around larger organizations that managed to adapt quickly because they had been preparing for the digital era. The focus of the study presented here adds the much-needed viewpoint of those individuals who form the target audiences and are vital for the future of any CCI organization. These are the results of a public survey aimed at informing directly cultural organizations that experiment with new creative approaches and are adjusting to the transformations that have taken place. Findings are vital for organizations from smaller cultural markets while key conclusions shed light on the new disposition of the public towards existing and future formats of cultural offers online that are valid for contemporary audiences in general. These cover several key observations regarding the most popular type of cultural content and events online; the engagement rate of different audience groups; the most preferred duration of culture in video format; gender affinities influencing the engagement with cultural content online; the motivations and barriers for attending cultural events online, ways to keep the audiences informed, and finally, which new forms of cultural and creative offers would the public be willing to attend online in the recent future. The article demonstrates that knowing the target audience and using data analytics on public dispositions while creating new content can empower CCI organizations. This claim is also based on the successful experience of pilot offers created by CCIs that have benefited directly from the research results of Intercultura Consult's 2021 project. Work continues in a new project empowering cultural organizations to incubate experimental offers (digital dimension including). The goal is to enhance their connection with key audiences.
\end{abstract}

\section{Keywords}

cultural participation online; digital demand; CCI innovation.

\section{Introduction}

The pandemic of COVID-19 while extremely challenging for the cultural sector, provided an opportunity for re-evaluation of the sector, the policies that guide it, and its impact on social and economic development. It, thus, brought forward an opportunity for change and evolution. Some CCIs jumped at the occasion to experiment and launch new services and creation, others were taken by surprise and left aside.

Culture's economic importance and the value of the creative industries for growth and jobs became once again a matter of debate. It was quickly obvious that not a single country should underrate their importance. In 2020 in France, PRODISS estimated that due to the COVID-19 crisis in two months, the industry would have lost 590 million euros (PRODISS, 2020). In Bulgaria, a country with a much smaller market, a survey of the sector demonstrated that the expected revenue losses from the crisis until July 2020, would have been around 8 million euro; or 21.5 million euro until the end of 2020 (Stoeva, 2020). These numbers define CCI professionals and their activities as an important economic driver. Advocates of the cultural sector also underlined the intrinsic values and potential of the CCIs to be "a major accelerator of social, societal and environmental transitions in Europe" (Lhermitte et al., 2021). The role of CCI's cross-

\section{How to cite}

Koleva, P., \& Berbenkova, M. (2021). Reconnect: Cultural Content and Audiences in the Digital

Environment. Culture. Society. Economy. Politics, 1(2), 12-31. https://doi.org/10.2478/csep-2021-

0008

ISSN (online) 2810-2010

https://website.sciendo.com/publication/culture-society-economy-politics/

http://www.facultateademanagement.ro/culture-sep/ 
sectoral innovation and its value in view of transformations that affect industrial and social change became a topic of international discussion (Koleva et al., 2021).

Above all, the re-valuation debate was dominated by the urgency of 'becoming digital' that affected/will affect all CCIs- irrespective of their location, type of activity, or state of readiness. This was the immediate factor driving innovation in CCI's creative processes and services that transformed the marketing and sales aspects but more than this. It also brought forth new factors defining and motivating cultural attendance such as the attention span of the public and new forms of engaging the public online. Indeed, digital innovation and social innovation have evolved as common priorities of CCI organizations (IDEA Consult, Amann, \& Heinsius, 2021).

Organizations and artists alike turned to the digital realm in need of finding a 'digital' outlet for their activities and of establishing a new connection to their audiences. Many of them were doing this for the first time. In Bulgaria, as in many parts of the world, CCI professionals had to use skills and tools previously unknown to them. This led to an outburst of digital cultural content that was seeking to engage the audience. The quality was unstable, some offers had great content and some others were less spectacular. More importantly, this was a time in which new creative forms appeared which the sector had previously not considered possible (Berbenkova \& Koleva, 2020).

Many of us who work in the CCI sector or are collaborating with cultural and creative professionals have by now formed a good idea of the new status quo. Valuable research studies have focused on the industry side and its needs. A lot of attention has been placed on the value of 'relief video content' and the fact that online films, audiobooks, and various digital art forms provided comfort to individuals in isolation. Research on CCI's influencing wellbeing became even more prominent. The spotlight has also been put on ways in which COVID-19 has increased the public interaction with virtual cultural heritage in European countries (Wiesand \& Ateca-Amestoy, 2021; Sofaer et al., 2021).

One may conclude that digital innovation and social innovation have become a dominant part of the cultural policy discourse over the last year. The concepts of externalities that CCIs deliver to society at large have progressively broadened the notion of CCI industries benefiting the economy and society (European Commission, 2010). What the authors found lacking in view of obtaining a '360-degree view' of the transformations that have taken place was the viewpoint of the public. The public, the audience, the consumers of cultural goods or services were the object of research. It was time to address the audiences and their perception of the CCI transformation particularly when it came to how cultural attendance and participation were affected. This important insight is, in the opinion of the authors, vital to creatives, cultural managers, or policymakers. It regards the analysis of the actual uptake of digital cultural content by audiences.

This is why, in August 2020, Intercultura Consult started working on a project that aimed to address precisely this issue. The project "Reconnect: Audiences and Cultural Content in the Digital Environment" consisted of a public survey on dispositions regarding cultural content online, four focus groups with representatives of the general public targeting specific cultural content (the general attitudes of the audience towards culture online, contemporary performance art, children's events, and cultural tourism). The special focus of this project, realized in partnership, was to create a bridge between the research of audiences and the current focus of CCI organizations working in the period of adaptations. The project was realized with the support of the National "Culture" Fund, Bulgaria through a dedicated program called 'Public' which offers a grant to do research on the public or specific audiences and to raise the capacity of cultural organizations (Intercultura Consult, 2020). 


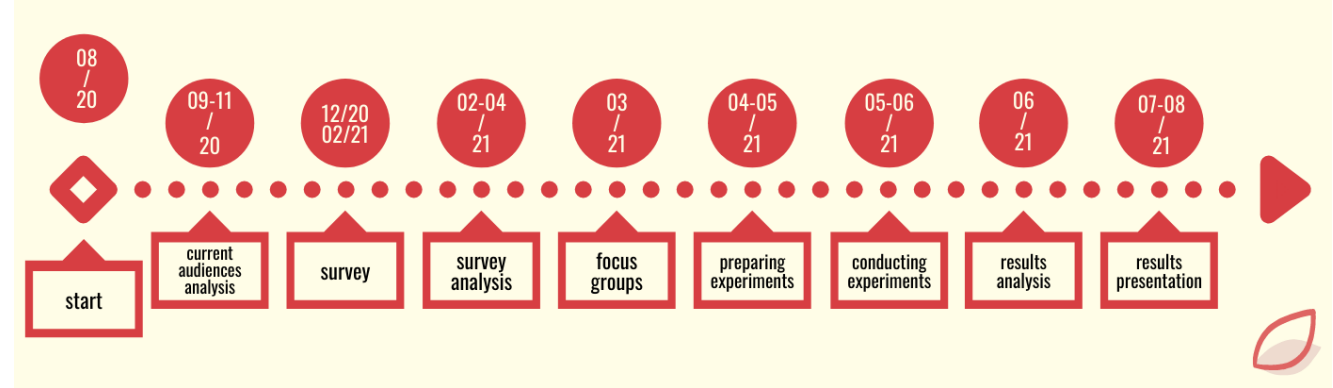

Figure 1. Infographic Reconnect: Audiences and Cultural Content in the Digital Environment (Intercultura Consult, 2020)

Besides research of the public, the project also integrated an incubation process involving three cultural organizations. The activities of support are aimed at two related goals: 1) for organizations to obtain new knowledge about their audiences and also of key public dispositions, and 2) test approaches that will be beneficial for organizations.

Each creative organization undertook experimentation with creating new cultural content based on new knowledge and in all three cases the results have been very positive. The project offered a unique opportunity to examine the disposition, expectations, and reaction of the public to new offers in Bulgaria, where data on audiences is rarely collected. There are only incidental research publications on a specific audience and target groups. Thus, the project allowed the partners to embed the research insights into creative and cultural marketing practices of the organizations, innovating the connection with the public directly and tangibly.

This article presents a selected focus on key results concerning the public primarily. It is divided into two parts focusing on 1) current dispositions of the public and 2) emerging preferences for cultural content online.

\section{Research concept and methodology}

The survey of the public was conducted online during a period of strict lockdown measures in Bulgaria and most of Europe, early in 2021. It aimed to research the attitude of members of the public towards the phenomenon of online cultural events and content.

One should bear in mind that the study focused primarily on the consumption of cultural participation aspect. To a much lesser extent has it discussed the supply side of culture (online and offline). This inevitably influences the results obtained, since cultural participation is in general considered to be led by the offer, not by demand (Koleva, 2013). To obtain another level of relevant conclusions, a comparison between supply and demand in each of the cultural sub-domains discussed would be required. This study had its limitations and provides a good opportunity for further work.

The survey was conducted mostly addressing the public in Bulgaria but there was also an English version of the same groups of questions collecting feedback from international respondents based in other countries. The survey did not address all forms of culture; individual artworks or content. It was completed by 714 people, of whom 502 answered all questions. 


\section{Limitations and validation}

The purpose of the survey and focus groups consultations was to reach out and engage as much as possible the wide public. These were defined to be people who are: 1) not cultural and creative organization professionals and 2) are potentially the audience less regularly engaging with cultural offers. The survey did not cover all forms of culture and did not aim to compare individual artistic disciplines of artworks. The survey was promoted using paid ads.

The results presented here are based on actual responses to a selected number of questions, any missing data are excluded. For instance, demographic data was not mandatory and responses were filled only by willing respondents, thus $70 \%$ have answered these questions. The analysis provides the unique view of those who completed the survey.

The profile of respondents includes $70 \%$ of professionals who do not work in the cultural and creative industries. A higher percentage of representation was found among respondents working in education, marketing, and communications, IT, finance and economics, design, and humanities / social sciences; over $19 \%$ of university students - including doctoral and employed students, $18.33 \%$ at the age of $19-25$, and $28.88 \%$ at the age of $26-35$; at least $35 \%$ of the respondents reside outside Sofia, Bulgaria, $7.62 \%$ outside the country. Some respondents did not share in which city in Bulgaria they live; $79 \%$ women and $18 \%$ men, additionally $3 \%$ offered no answer. Some of the survey results have been further validated via four focus groups engaging 43 persons conducted as a second research step in the project.

\section{Public preferences in the usage of cultural content online}

The findings presented in the first section are based on analysis of the data collected via the public survey and enriched with additional analysis via focus groups that were carried out during the project. The findings can be divided into seven topics:

- audiences for online and offline cultural content: engagement rates;

- most used cultural content online and preferred length;

- $\quad$ engagement with culture: online and offline;

- gender affinities influencing preferences for cultural content online;

- motivations and barriers for attending cultural events online vs. offline;

- information channels about cultural content;

- emerging preferences for cultural content online.

The first most immediate goal of the project was to attempt a mapping of who are the audiences and what are their usual ways of experiencing cultural offers. This resulted in a comparative view of preferences for in situ versus online attendance. The period of conducting the survey at the end of 2020 and the beginning of 2021 was seen as the right moment for people to have a clear memory of both past habits and new practices. We expect that by the end of 2022 memories to be blurred, so the profiling obtained here is, in a certain sense, unique.

\section{Audiences for online and offline cultural content: engagement rates}

In general, by frequency of attending cultural events, audiences can be divided into three large groups. There are: Active - attending once a week and more often; Potential attending a few times per month (but less than once a week); Passive - attending less than once a month. 


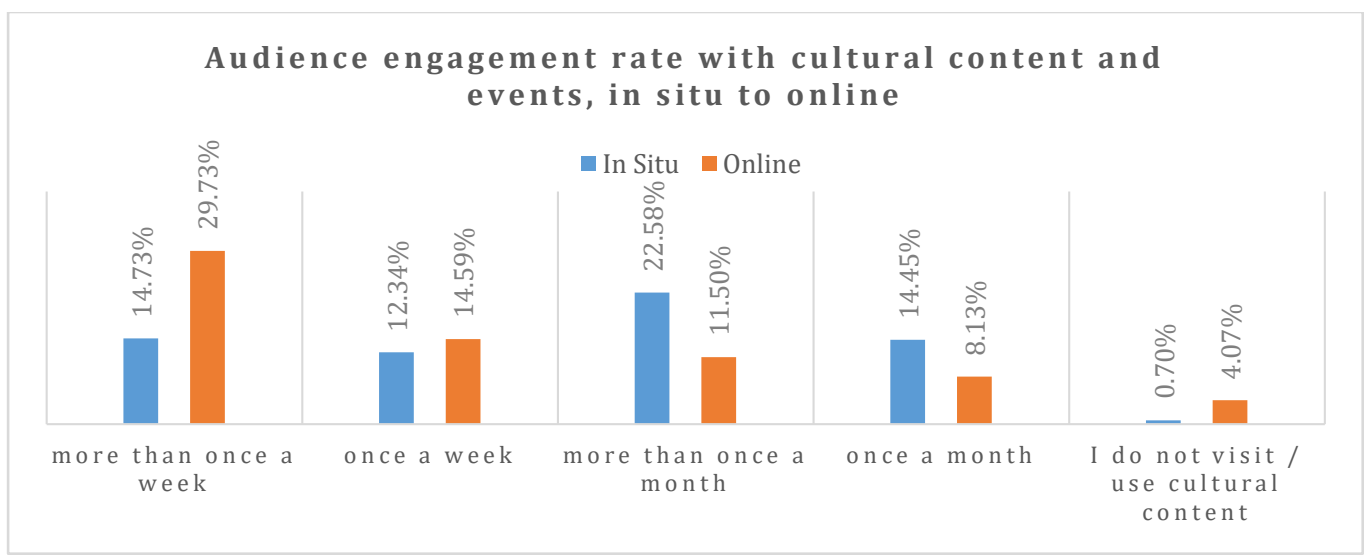

Figure 2. Audience engagement rate with cultural content and events - in situ to online

Unsurprisingly, the responses confirm that active audiences for in situ events are also the most active users of online cultural content. By contrast, the 'potential' and 'passive' groups of audiences of in situ events tend to decrease their level of engagement with cultural content online, as seen in Figure 2 above.

One can easily conclude that online consumption exceeds the in-situ one, potentially driven by the increasingly diverse offer and greater flexibility of the supply side which allows live streaming, on-demand, or hybrid forms of interaction. However, looking closer at the data, a disturbing trend is also prominent. In the context of a pandemic and a predominant online supply, the less engaged public is weakening their engagement with cultural content.

\section{Most used cultural content online and preferred lengths}

In line with observations of the marketing and social media industry, the respondents indicate video as being most frequently used (45.2\%). The reason is that the medium of video, using moving images as well as sound is more engaging and possibly captures more of the authentic cultural experience. It can be used by cultural operators to attract audiences in a meaningful way. One key contribution of this study is the finding that the preferred video length according to the public is longer than one is led to believe.

Indeed, marketing experts claim that in the digital environment, a person has a shorter concentration span than a goldfish (9 seconds) (McSpadden. 2015). As a rule, digital marketing gurus recommend using videos of length up to 30 seconds and grabbing content duration of 3 seconds. In the present study, however, videos of cultural content lasting up to 20 or even 40 minutes are preferred by the public more than those of up to 5 minutes duration.

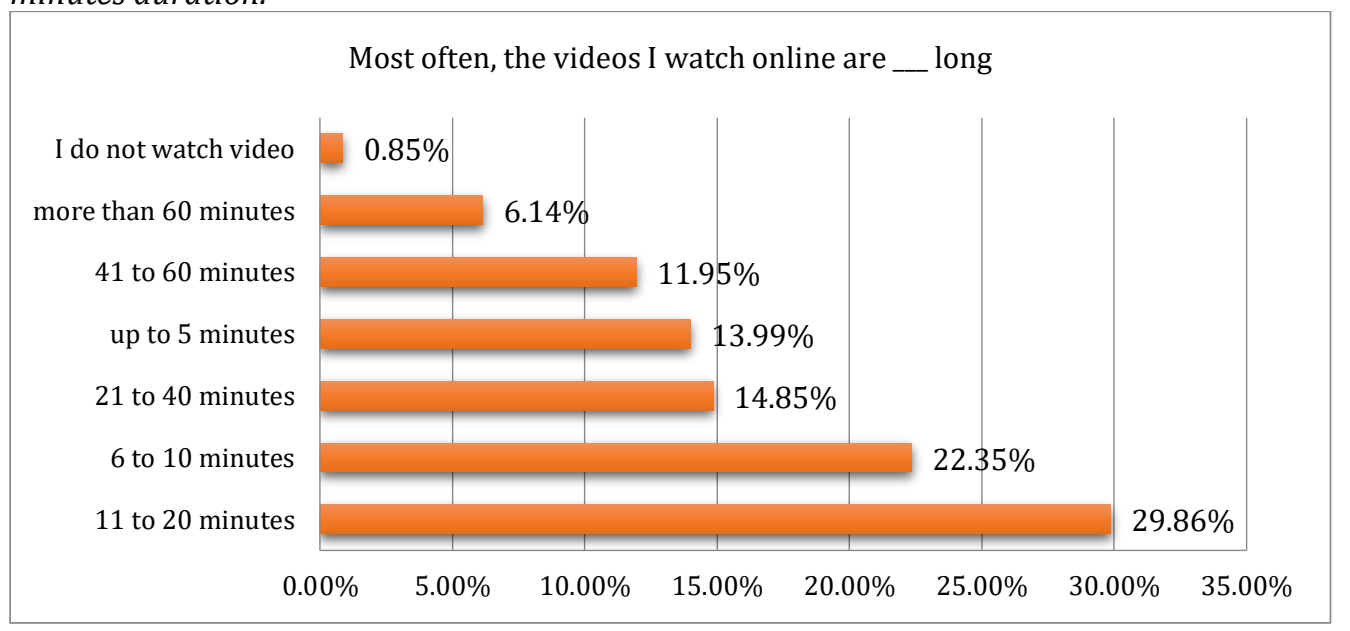

Figure 3. Preferred length of content in video format 
Respondents indicate that the videos they watch online usually last between 11 and 20 minutes, as visible in Figure 3. In interpreting these results the following additional observations should be taken into account:

- Short video length is usually associated with the preferences of audiences younger than 25, while the largest part of the respondents in the survey is over 25 years old. In this study, the age group expressing the strongest preference for shorter videos (6 to 10 minutes) is 46 - 55-year-old. This finding suggests that preference for long video content is correlated with other determinants.

- One of those important factors that drive the interest in longer video content is the habits of cultural participation cultivated in the public. These are formed over time of cultural exposure and are associated with the capacity of audiences to maintain long-term concentration to follow the intricacies of a plot, the abilities to transfer attention from detail to abstraction, etc. These individual dispositions and capacities are also collectively developed over time and may be subject to new patterns which are the reason the study also asked the respondents to set some projections for the future, (see Emerging preferences for cultural content online).

- Finally, one should not underestimate the fact that respondents to the survey were experiencing a state of lockdown (due to the pandemic) at the time of sharing their views. They were confined at home and, thus, more inclined to dedicate time to consuming cultural content online. The regular routines of most respondents in 'normal' pre-pandemic circumstances are likely to have been defined by different factors such as time pressure. Under 'normal' circumstances such time constraints would have imposed limits to the duration of videos one would choose to see as a means for cultural participation.

\section{Engagement with culture: online and offline}

As seen above, cultural content engagement online is generally stronger than the one offline. This is valid for rather different types of cultural content, showcasing correlations and dissonances between online and offline cultural participation in specific situations, see Figure 4.

The overall cultural engagement online is stronger when compared to "live" attendance for the art forms listed in the survey filled by the respondents. This trend can be explained by the immediate action undertaken by the CCI sector which resulted in offering a more even supply of various art and culture forms online. These digital offers overcome (more easily) geographical and financial barriers. These conclusions are supported by the other data in the survey presented in section Motivations and barriers for attending cultural events online vs. offline. In addition, speaking of Bulgaria, it is worth pointing out that in general, access to good internet is available even in places where there is normally next to nothing in terms of cultural offer.

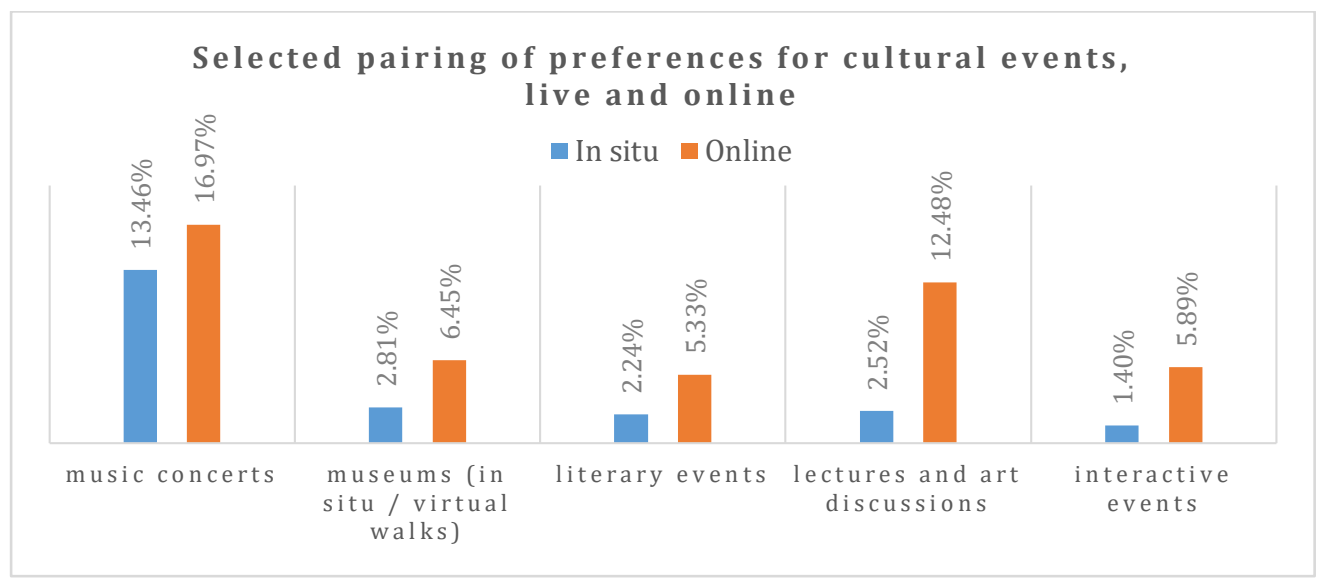

Figure 4. The selected pairing of preferences for cultural events - live and online 
The respondents have rated the preferences shown in Figure 4 above in two separate questions for their 'habitual' in situ- and for their 'current' online usage. These questions were set apart from each other in the survey, so they are likely to reflect an authentic reaction. Music concerts and theatre performances (not visualized in the chart above) take the first two places in audiences' preferences towards cultural events for both options of attendance - digitally or on location. However, the preference for attending physically events in the theatre is considerably stronger than that for the same art for online forms of attendance (26.23\% compared to $15.57 \%$ online), while online consumption of music concerts outweighs the regularity of physical attendance $(16.97 \%$ online, $13.46 \%$ live). The interpretation of these results is further discussed in the section Motivations and barriers for attending cultural events online vs. offline.

Virtual museum visits and tours are more popular compared to their in-situ counterparts ( $2.81 \%$ live by $6.45 \%$ online). This higher interest is closely correlated to the increased offer of (free) online content from foreign and well-known institutions online, presented in high digital quality offering an interactive 'online visit', e.g. Le Louvre. Bulgarian museum institutions did not propose many online activities at the time of the survey and did not obtain the same level of engagement from audiences online. This important finding was further confirmed in discussions in the focus groups after the survey had been completed.

While museums attendance online is increasing in general, the visits to art galleries online fare less well. This is likely associated with the same trend of relatively low supply by Bulgarian galleries at the time of the survey. One more fact should be considered. The galleries, more than museums, are affected by the art market in general, particularly collectors. Even in Bulgaria, where many art galleries are institutions supported by state or municipal funding, this is the case. This is why, the findings that 'one-third of businesses downsized during the first half of 2020' and some faced closure because they lost staff would be relevant to Bulgaria as well (McAndrew, 2020). This said, public support for the private 'for-profit sector as well as for the 'free/independent/not for profit arts organizations, has become an open debate and priority since 2020 in Bulgaria as well as in many other countries of the EU. As a result, some reforms have been implemented of the support measures for CCIs, in view of revaluation of the positive contribution CCIs make to the economy and society. New programs have opened at a national and local level and legislative changes are discussed which may be seen as a positive side-effect of this period of hardship. While we expect enforced policy measures in the coming period which would protect the rights of arts and cultural professionals, it is clear that the online offer is diversifying and increasing at a very rapid speed.

By the time of writing this article, the authors are noticing the rising awareness of galleries and other organizations of the need to engage with their audiences online. A striking preference for online attendance over the in-situ attendance regards the lectures or art discussions $(12.48 \%$ online, live are $2.52 \%)$. This is a very important trend that should be taken into account by cultural operators as it regards the increased demand for educational content along with the core online offer or service.

Since video is the most preferred form of cultural content online ( $45.2 \%$ of respondents) it is reasonable to pay attention to the arts most closely associated with it: cinema and films. In terms of physical attendance, cinema ranked as the top preference by audiences. The survey did not explicitly ask respondents to express preferences towards its online equivalent, as the answer was likely to skew the responses. However, respondents who had selected the option "other" to the question asking them to indicate the most preferred form of online culture, most often reported the attendance of online "movies" and "film festivals". These preferences were further confirmed by the focus groups where the interest in film festivals, movies, and series proved to be higher than the one in online performing arts (music concerts, theatre, etc.). Once again, the state of forced isolation is to be considered a factor stimulating the increase of film consumption online. 
The study found that the least attended events, both online and offline, fall in the categories: classical ballet and opera, interactive events, contemporary dance performances, and literature. Once more, access has to be considered as one of the factors influencing these results. For example, in Bulgaria, opera performances are mainly held in the capital. However, for all these genres, the study confirmed that online audiences are more active than offline ones.

\section{Gender affinities influencing preferences for cultural content online}

While both men and women generally demonstrate the same preferences towards cultural participation in situ and online, some affinities became clear in the analysis.

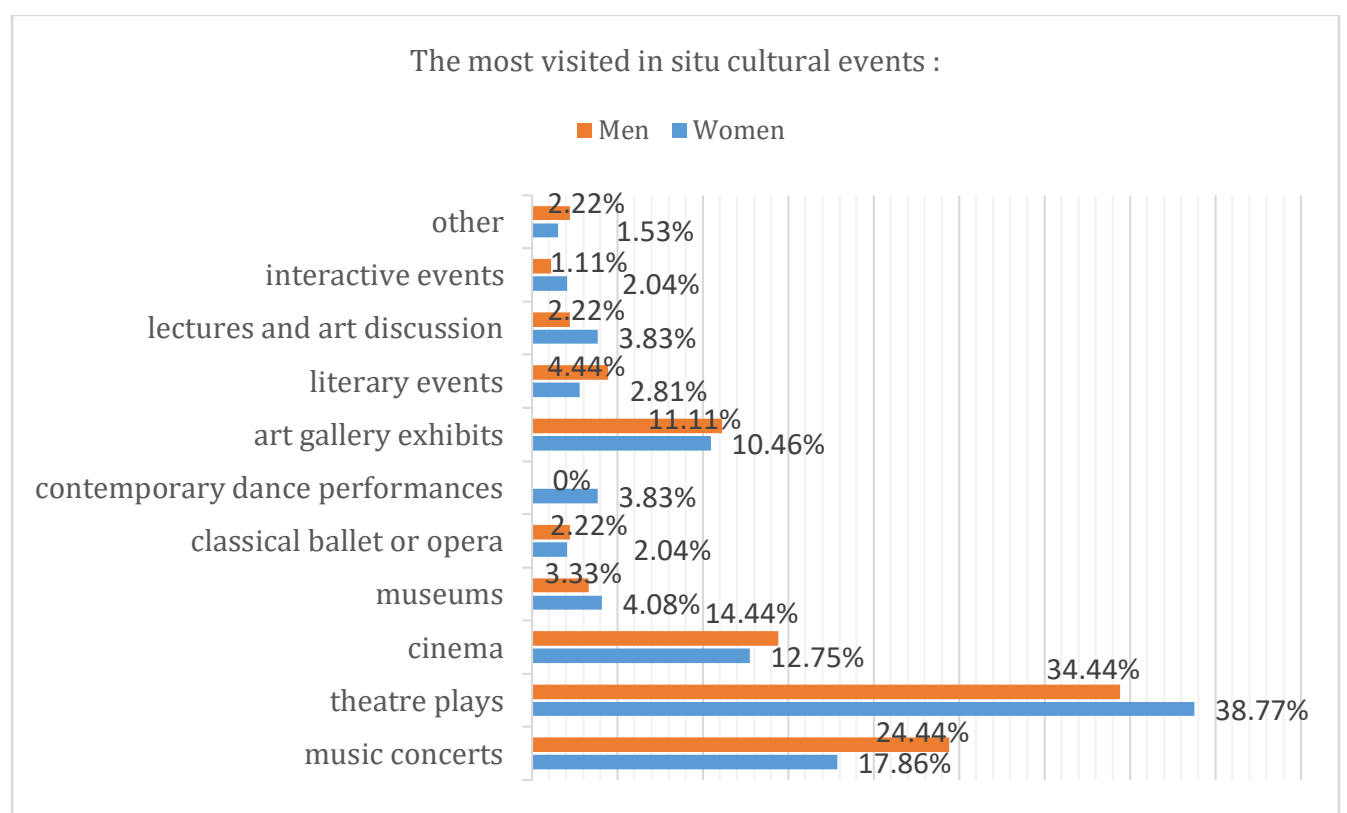

Figure 5. Comparison between men and women in the preferences towards live cultural events

As seen in Figure 5, theatre plays are the number one choice for in situ events for both groups, yet women express a more pronounced interest in attending theatre events than men. Musical concerts as the second choice of preferences, capture more firmly the attention of men. When it comes to classical ballet, literary events, and interactive events, there is no significant difference between men and women.

It is noteworthy that none of the male respondents indicated contemporary dance performances as a favorite event in situ while $3.83 \%$ of women have expressed such a preference. 


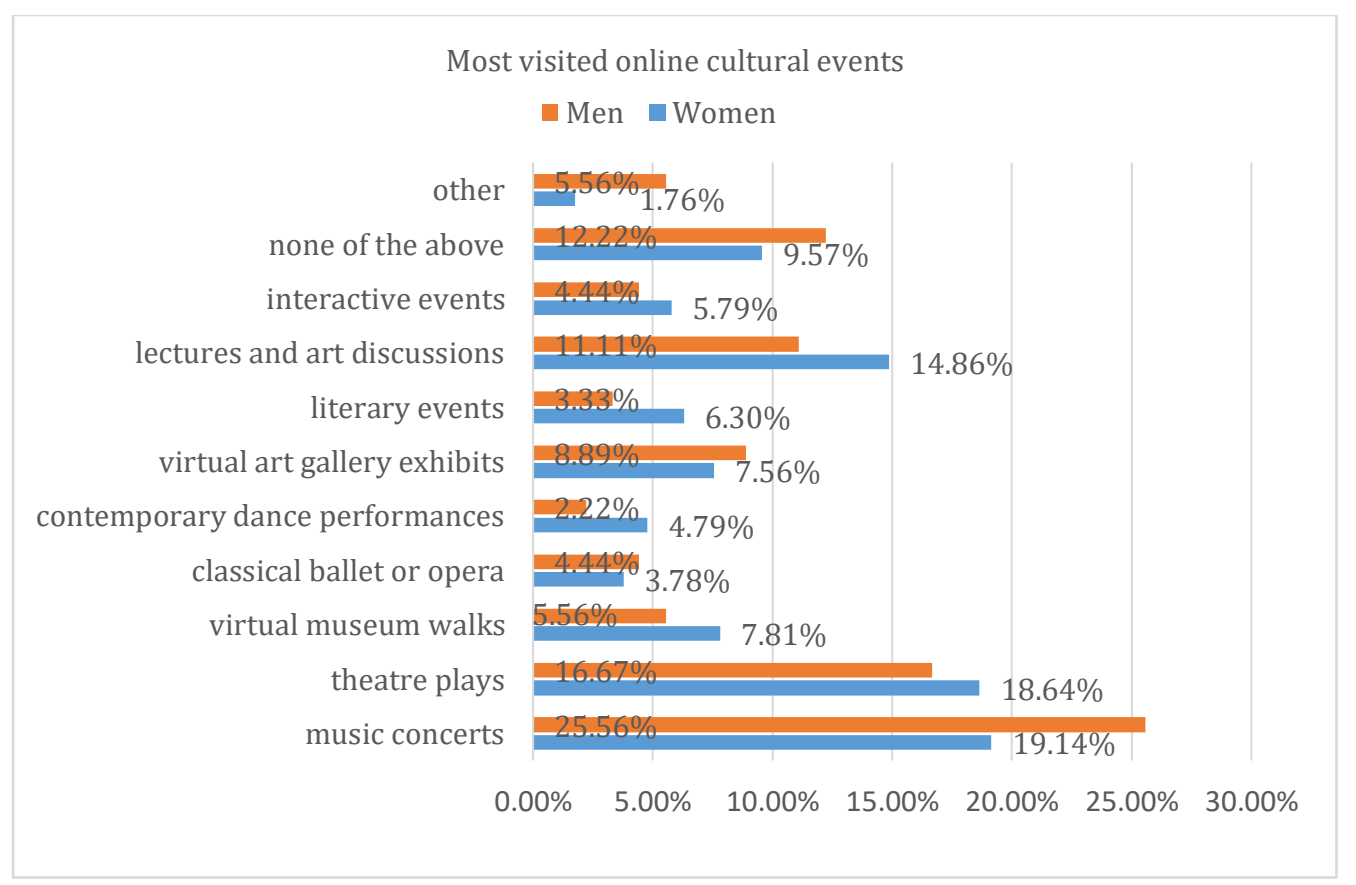

Figure 6. Comparison between men and women in the preferences towards online cultural events

Online, the affinities remain similar. There is a more even distribution in the preferences expressed for the various arts online that is impressive. A factor contributing to this trend is the seamless way in which additional information about the arts form or specific event or cultural offer is available and actively demanded by both men and women in the audience (discussed in section Engagement with culture: online and offline above). It is very likely that cultural organizations accompany their offer with promotional content which stimulates interest.

The main difference by gender affinity is that online there is a higher percentage of men demonstrating interest in modern dance. A possible explanation is the newly discovered availability of performances that are becoming more accessible online. Another factor discussed elsewhere in this article is the anonymity and freedom to explore associated with online consumption. This information may be useful by contemporary dance companies and organizations to attract new, diverse audiences.

\section{Motivations and barriers for attending cultural events online vs. offline}

The aims of the study were not to validate specific assumptions around cultural participation based on theoretical projection. The project was designed on the premise of fact-finding regarding the dispositions of the public at a specific moment in time. Its goal was to obtain new data regarding some of the well-known factors defining a potential cause for audiences to engage often and actively with cultural content online. 


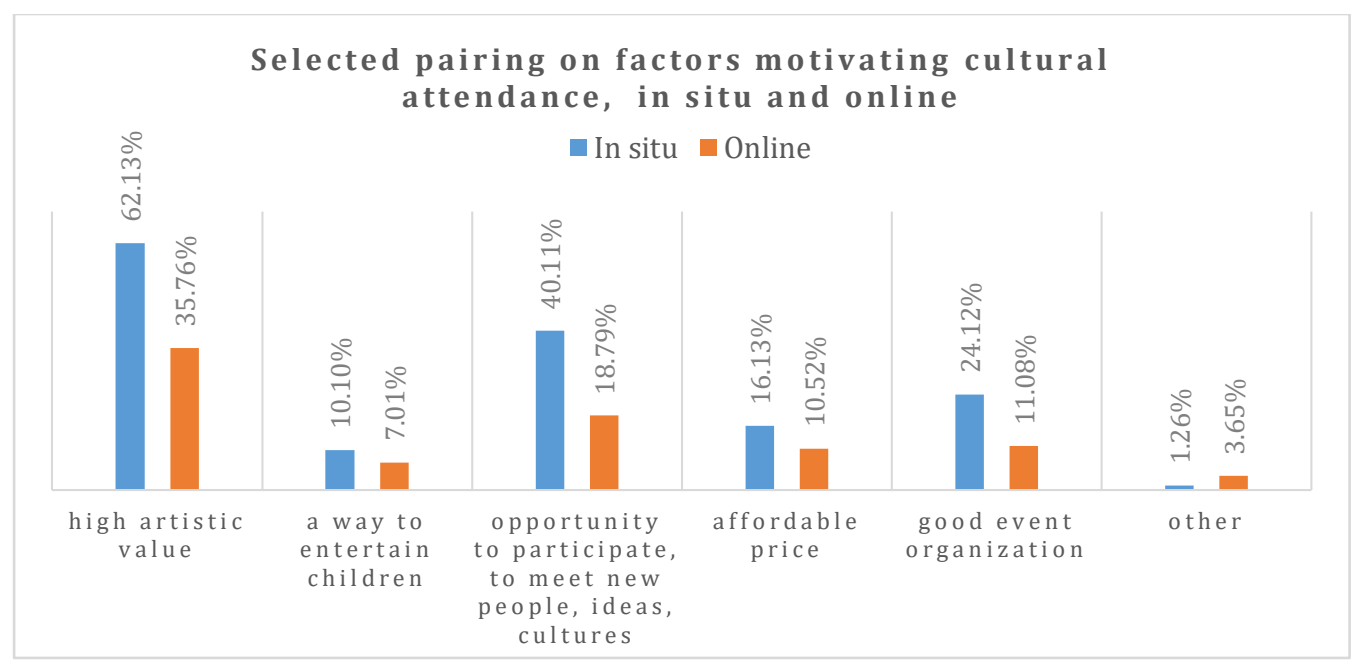

Figure 7. Selected pairing on factors motivating cultural attendance - in situ and online

Among the most powerful motives for attending cultural events in situ are the socialization aspects such as the "opportunity to participate, meet new people, ideas, and cultures". This is a response selected by the respondents at $40.11 \%$ for live events vs. $18.79 \%$ for online. Excluded from the pairs in the above chart is the data on events in situ for which the highest-rated motivation to attend is "the opportunity to meet friends and colleagues" at (42.22\%). Since this optional answer was not directly relevant for 'online' cultural attendance at the time of the survey, it was not presented.

This factor, in combination with the stated interest in lectures and discussions about art online, showcases the need for CCI organizations to consider ways to meet this demand for opportunities for social interaction (discussions, forums, surveys, etc.) while presenting products online, thus compensating at least in part the loss of social interaction.

By contrast, respondents value the online opportunity to participate in events that are otherwise unavailable (54.98\%). This high response rate is certainly influenced by the situation of the lockdown with no access to public events. Additionally, as confirmed by the focus groups, online attendance of this choice regards events that take place in remote places, that are inaccessible financially or geographically, and particularly events by international stars and organizations that rarely tour in Bulgaria. In this regard, the finding indicates that a future study may need to compare public demand with the supply side (online and offline) so that even more precise conclusions can be drawn with respect to specific arts or cultural productions and content.

The motivation to engage with "high artistic value" is cited as most relevant to both online and live attendance. While it is the leading motive in both scenarios, there is a significant priority for in situ culture $(62.13 \%$ vs. $35.76 \%$ online). The decrease of importance for online participation may be because in the digital environment the audiences are more willing to experiment in selectively trying out new content since the risks of a wasted financial investment or the social phenomenon of not 'losing face' are less pronounced.

The motivation to attend a cultural event to accompany children did not emerge among the top choices selected by the respondents. The response rates are comparable for online and offline use with a slight advantage for offline offers $(10.10 \%$ compared to $7.01 \%$ online). One reason is that a larger group of respondents in the survey are likely to have been people with no children or with grown-up children (per estimated age groups) and this affected the response rates. In one of the focus groups, however, where parents and teachers discussed the existing online cultural offer for children, it became clear that both groups are actively engaged with seeking and providing cultural experiences to children. Parents expressed concern with the difficulty of obtaining 
information about suitable events for children as well as in finding cultural content in Bulgarian.

As seen in Figure 7, affordable price (16.13\% live vs. 10.52\% online) and good event organization ( $24.12 \%$ live vs. $11.08 \%$ online) are rated higher for live events than online. This is a sign to the organizers of online events to rethink the organizational approach and pricing policies. The readiness to pay online is lower and is correlated with expectations for improving the quality of the offered events (in terms of content and technology).

Among the key motivators for acquiring 'cultural capital' is the fact that it embellishes the position of the respective individual among a distinctive group in society (Bourdieu, 1987). The data presented here proves once again that the social vector of cultural participation is very important to the audiences and it plays a significant role in several dimensions. It may present a barrier to online participation as discussed above because it is a factor informing the choice of cultural offers (discussed in section Information channels about cultural content) and it drives emerging preferences for online engagement (analyzed in section Emerging preferences for cultural content online).

A contribution of the study is also the finding that online practices may be motivated by a related human desire of being 'invisible'. Online participation offers a chance of sometimes being less exposed to the judgment of others regarding the cultural taste we demonstrate.

Finally, a considerable incentive for online culture participation in Bulgaria was the lifting of (financial and physical) barriers to attending an event of interest at the given period.

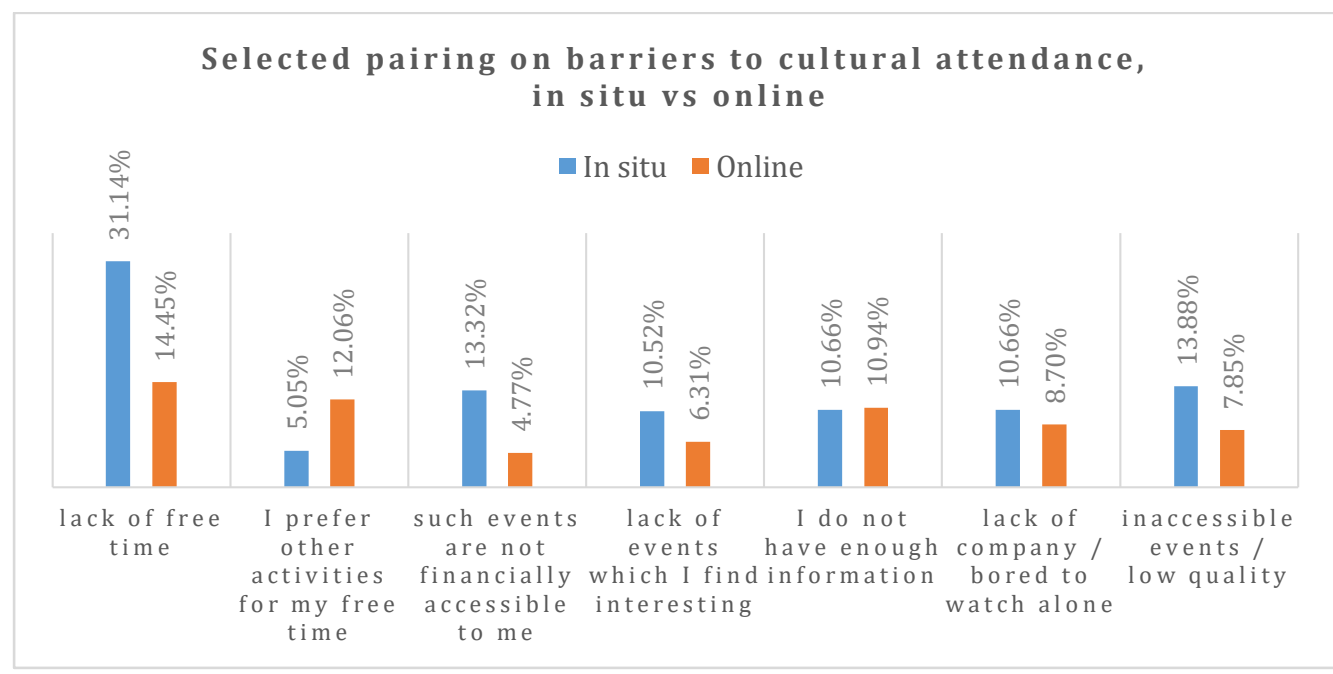

Figure 8. Selected pairing on barriers to cultural attendance - in situ vs online

According to the survey data, the most serious barrier to the consumption of cultural production, both live and online, is the lack of free time (31.14\% live $14.45 \%$ online). This barrier is significantly higher for live visits. This is understandable, as they demand more time from the visitor to prepare, travel, organize care for dependent children, etc. Cultural operators working on online content would benefit from expanding and diversifying their offer online in view of this trend as online attendance requires less effort. They, however, should consider another factor, the time of cultural engagement being flexible, leaving room for a personal choice on when to attend online events.

While the remoteness of the locations is ranked as the second most commonly recognized barrier to attending live events (13.88\%), the indirect equivalent to this barrier in the responses regarding online culture is associated with the dissatisfaction 
with the technical quality of the events. Some respondents indicate this as a barrier to online cultural participation that may be interpreted as distancing them from the experience of a live event, (7.85\%).

The second most important diversion from engaging with online cultural content is alternative leisure time preferences according to $12.06 \%$ respondents for online and $5.05 \%$ live events. The situation of confinement and restrictions evidently provides more choices for recreation besides culture. Additionally, in the context of forced isolation, people spend more time online to accomplish various other activities which limits their capacity to absorb online content. They work, study, and even connect with friends and relatives online which often leads to digital fatigue (Auxier \& Silverglate, 2021). In this sense, further research is needed on the structure of leisure time in a pandemic situation, to be compared with the 'usual' activities and forms of cultural participation. Digital fatigue presents an important new barrier to cultural participation.

As expected, the financial barrier is less pronounced with regard to online events (13.32\% live versus $4.47 \%$ online). These results are influenced by the wide affordability of online cultural events, which were offered free of charge especially in the first months of the pandemic.

The only barrier rated of equal importance for both online and offline cultural participation (about 11\% of respondents) is no 'information' about events. The perceived lack of suitable offers defines another obstacle for the attendance of a considerable share of those attending live- or online events, $10.52 \%$ and $-6.31 \%$ respectively. The absence of territorial/geographical barriers to attending online events is a problem for fewer people, yet the share of those unaware of suitable cultural offers online is considerable. Thus, one may conclude that using the internet to provide cultural access to more diverse events would benefit both organizations and audiences. Yet, there is still a problem in reaching specific target audiences.

\section{Staying informed about cultural events}

When asked about their awareness of suitable cultural events, $10.94 \%$ of respondents state they did not have enough information about relevant online cultural events. This result testifies of a gap between the efforts of cultural operators to reach new audiences online and the result. This is supported by findings in a focus group in which parents shared that they have limited access to information about digital events for children. This gap further underlines the fact that cultural operators need professional support to promote creative or cultural content. They would need to also obtain new marketing skills, as well as to improve communication strategies in general because they are the primary source of reliable online information.

A large share of the respondents $(28.19 \%)$ report to be consulting the "sites of cultural organizations", as well as (12.90\%) of "cultural guides and directories". This result suggests there is an active audience for culture, which takes initiative in staying informed about cultural events. This potential public is also aware of the official, most trustworthy sources of information in the age of (mis)information.

As visible in Figure 9, a high percentage of people report on being informed by outdoor advertising - posters, billboards, flyers, etc., as well as by the media. These channels are very important and, even if more expensive, they remain valid for promoting online cultural participation too. 


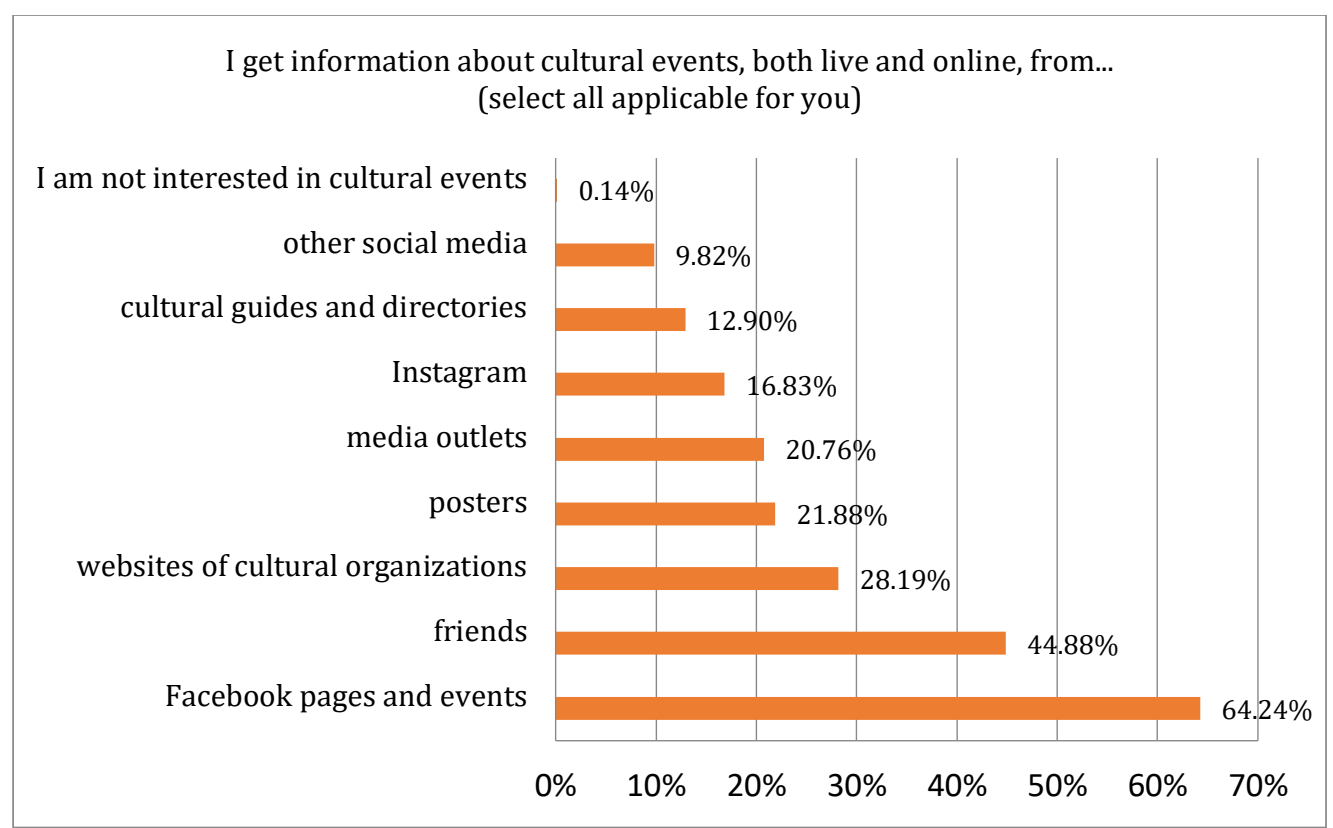

Figure 9. Staying informed about cultural events online and in situ from (multiple choice)

The general conclusion of this section is that there is potential for building a more effective and focused connection between culture and online audiences. Its evolution will depend on improving the digital capacity and infrastructure of artists and organizations. First of all, they need to develop the skills and find new means to attract online audiences via digital marketing and using a creative approach to promote their presence in the digital environment. It is important to maintain dialogue with the audience and use official channels as well as offline media for this purpose. Secondly, specialists who can assist CCIs in developing a communication approach relevant to the field of art and culture and at an affordable price have to be engaged. Last but not least, specific online platforms or digital media outlets have to be developed to provide CCI organizations of various sizes, types, and disciplines to inform their potential audiences via coordinated promotional activities, e.g. at the level of a given city or region (Lorenzen \& Friederiksen, 2007).

Advances will inevitably depend on CCIs acquiring skills to manage new tools or to outsource needs effectively. The last option would allow the smallest representatives of the sector to be less burdened by non-creative work and it is public funding that should address these new needs. The largest CCIs are already able to hire in-house staff or contract support on a longer-term basis but in any technological transition period, they also need public support. In any case, the sector will need to be given the opportunity to break open the boundaries of social network marketing, whose algorithms retrace the previous (non-) actions of a given person and a close circle of friends, thus limiting the choice of cultural offers being visible/accessible to the respective public.

Another avenue to improve connections with audiences online would be to explore possible new formats of cultural offers online that they are already experimenting with or willing to engage with. This topic was explored in the survey by inviting respondents to share potential future interests, even if they could not yet testify of a relevant experience. 


\section{Emerging preferences for cultural content online}

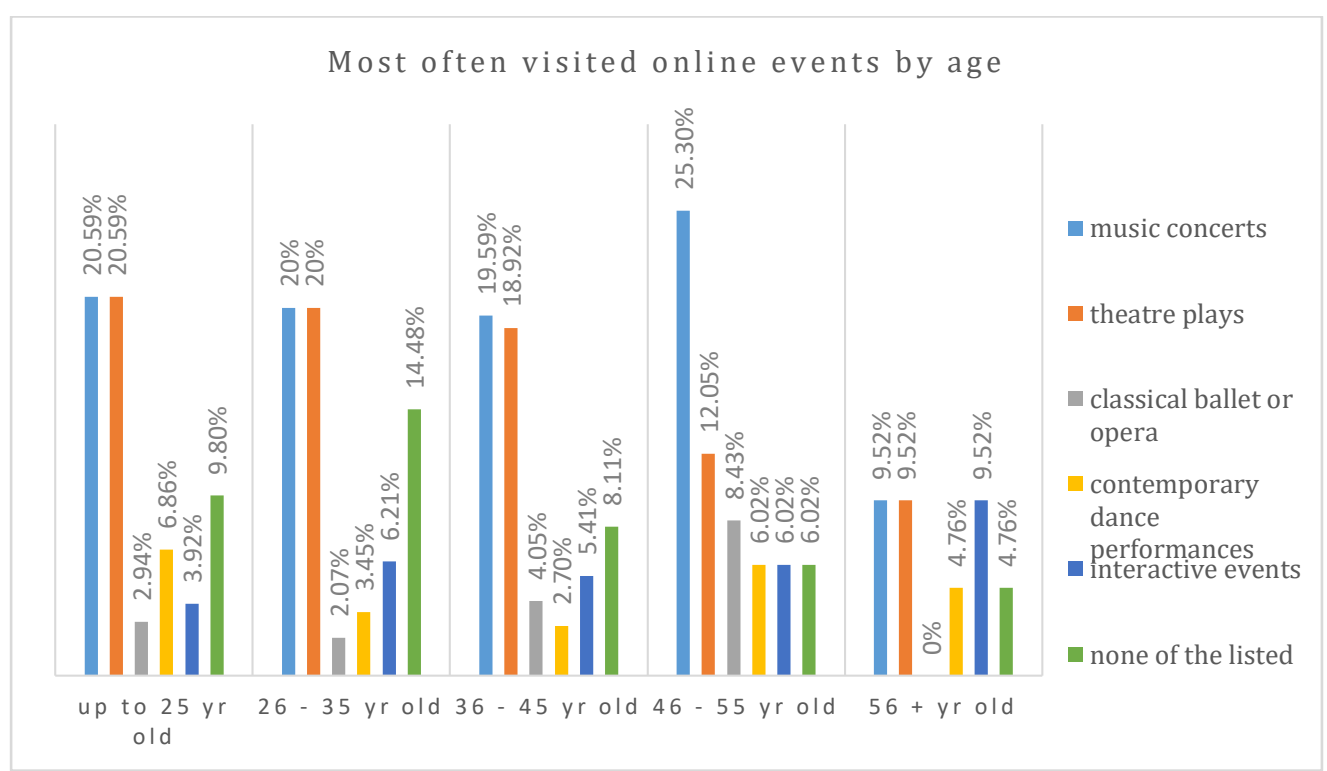

Figure 10 A. Most often visited online events by age

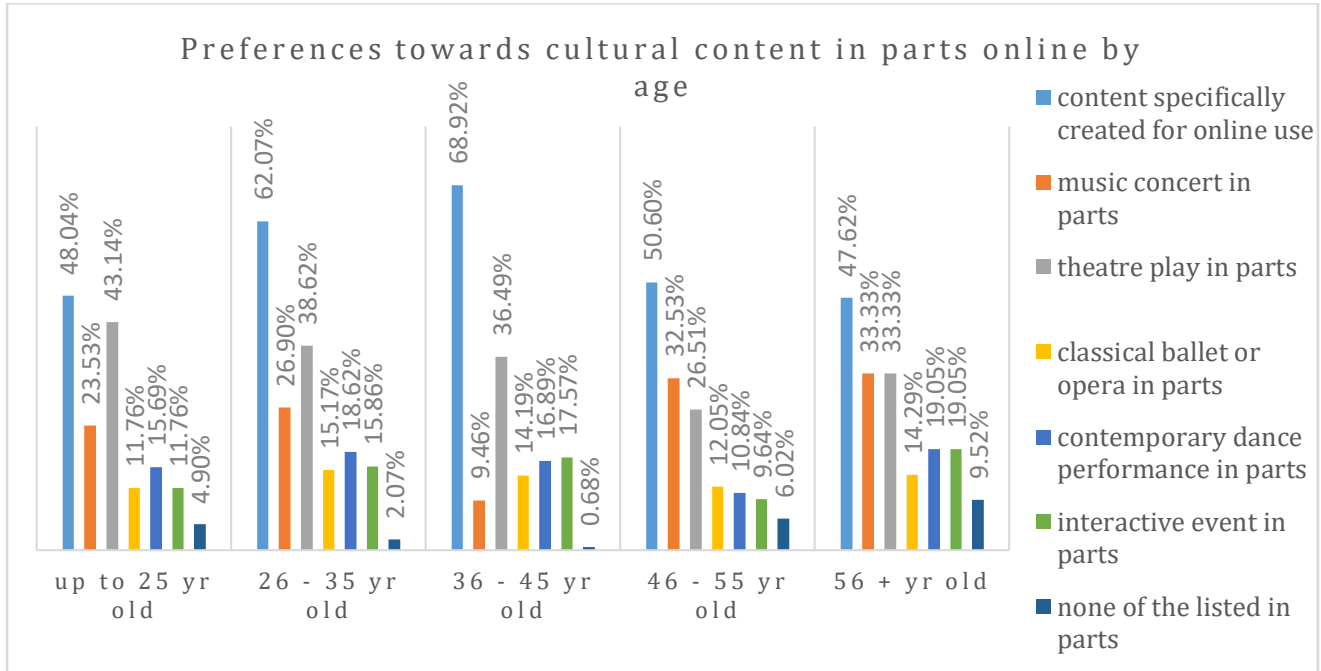

Figure 10 B. Preferences towards cultural content in parts online by age

Figure 10A and Figure 10B present the preference for engaging with cultural content "in episodes". All age groups have selected as the top choice the "specially created online content". This indicates a possibility for a performance recorded in episodes to provoke the interest of people who have previously not expressed interest in the respective content. One should bear in mind in reading this answer that the question requested one choice on the "most visited" type of events from the respondents, while the question on being "interested" in events in parts or "not interested" allowed for multiple answers. This has certainly affected the results but the conclusion is clearly in favor of experimenting with the new format.

As showcased in section Engagement with culture: online and offline, all age groups would normally prefer to attend theatre performances in situ. In Figure 10B, however, there is a clearly demonstrated interest in "theatre production" being presented in parts for the younger groups (up to 25 and up to 35 years of age). This preference is twice as high as that expressed in another response regarding the already available online theatre offer. There the age group up to 25 years old in favor constituted $20 \%$ while the 
same group selecting online theatre in series is as high as $43 \%$. Those aged 26 to 35 yrs. respectively favored online theatre at $20 \%$ but $38.62 \%$ consider the theatre offer 'in episodes' to be more appealing.

Even more striking is the fact that the older audiences who express less pronounced readiness to engage with cultural forms online, such as the people aged $56+$ showcase interest in online theatre being presented as a sequence of video-based content. Their interest increased more than threefold from $9.53 \%$ for full-length formats to $33.33 \%$ for ones delivered in parts. Similarly, the interest in a "music concert" being presented online in parts is more likely to be higher than the currently expressed choice to attend an entire event of the same type for the groups 46-55 and 56+.

There is also a significantly higher interest in the segmented offer of an online experience of arts such as "classical ballet or opera" and "contemporary dance performance" among those groups who have indicated zero to very little interest in engaging with the full-length recording or streaming of content of the same arts in the currently available online offers. For example, while none of the respondents in the age group 56+ have selected "classical opera or ballet" as a preferred type of online cultural content, however in episodes, this art form attracts more than $14 \%$. For the younger age groups (up to 25 and 26 - 35) the interest grows from $2-3 \%$ to over $15 \%$. These findings are a signal to organizations working with these typically more exclusive arts to reconsider their digital presence to attract new interest online.

In this section of the survey, respondents were also asked to express their views on an "interactive event in which I can participate" in episodes. This was more attractive for the three groups younger than 45, who had not assigned it any significance when defining their recent online consumption. The authors expect that this demand will grow as especially the younger audiences become accustomed to mixing and merging online and offline socializing behavior by 2023.

One may conclude that interest in full-length cultural online offers will expand but not as quickly as the engagement with the same content is likely to grow when offered in episodes. This assumption is further supported by the share of respondents in the groups up to 45 years old who define their current use of culture online to include "none of the above, options. Its peak was in the age group $25-36$ at $14.48 \%$; while in the same group only $6 \%$ selected the same answer for the option of engaging with the same content that would be offered in episodes. The age groups $46+$ who are even less interested in any long duration of culture online offers also prefer the same in episodes.

Correlating these results with the previously discussed findings there is ground for making several general recommendations to cultural operators working with online audiences. They should, first of all, provide more and better diversity of high-quality content online. Secondly, consider including offers divided into episodes. Also, create opportunities for interactive events allowing various ways of involving the audience. The last would also include educational events providing additional information about the work, the performers, the context of its origin, etc. Finally, rethink the pricing policy and the possibilities for presenting paid events. The willingness to pay for online culture is lower, but the potential audience is much wider and less inhibited by geographical, social, and psychological barriers.

\section{Conclusions}

The COVID-19 pandemic has presented new challenges such as the ad hoc closure of operations for many CCI organizations and considerable shrinking of staff. This also exacerbated disparities in the cultural participation of disadvantaged groups. However, the crisis also paved the way to new opportunities such as new creative processes and new means of communicating a cultural message. 
In terms of connecting arts with audiences, it accelerated digitalization and brought advancements that would have otherwise taken years. Our study revealed that the audiences were eager to engage with culture online within a few months of the enforced confinement, and a key finding is that the duration of time they are willing to invest is significant, up to 20 or even 40 minutes per interaction.

Some cultural offers easily translate into high levels of engagement online compared to physical attendance. Music events, museum visits, and film festivals stand out.

Another important finding is that the online attendance for lectures and art discussions is more appealing to audiences than the same offer in situ; moreover, this is true for young generations too. One more valuable contribution of the study is confirming that cultural dispositions by gender and age may be different online and there is a way to expand cultural horizons.

The main factors that influence the will and ability of people to attend cultural events online also differ considerably. This finding demonstrates that data analytics and research of public dispositions are and will be important not only for policymakers but also for the organizations themselves. This is exemplified by findings regarding expectations of technical quality online. It needs to be of a different dimension and reflect closely the current and emerging needs of audience groups, including being approached by tailored communication strategies.

What will the future look like after this initial state of urgent transformation and adaptation? There is the question of whether these processes will continue, or the CCIs will revert to regular activities. The study concludes that the public demonstrates a clear preference towards live, in situ, cultural participation for the same reasons as in previous periods of cultural media evolution. The benefits of experiencing culture they bring to the individual as being part of a community are valued high. Also, the intrinsic value that the arts/cultural participation brings to each member of the audience is vital for the public. Thus, the long-established fact that arts and culture bring positive externalities holds true in the digital age (Brookes, 2008).

Results also indicate that some digitalization trends are here to stay and CCI organizations will experience their transformative power. Several key conclusions relevant to CCI organizations and professionals should be stressed. Of primary importance, the study found that the presence (or lack) of information about cultural events is a factor that presents a serious barrier to both online and offline consumption. The study demonstrates that even in the age of information a high percentage of people (almost $11 \%$ of the respondents) testify of insufficient awareness of cultural events. Mass- and also misinformation plays a key role as a barrier to cultural participation. The gravity of this factor is supported by a good share of residents in the capital of Bulgaria who took part in the survey and focus groups and demonstrated sufficient interest and financial capacity to engage with arts and culture. This is a manifestation of a public need to be met via targeted efforts on the part of cultural organizations but the updating of approaches to connect with the audience should be supported by policymakers.

CCIs would need to widen their digital access and employ diverse information strategies to assist the public launch of any cultural offer (artworks/cultural events). Furthermore, the finding that "passive" audiences tend to be even less accessible online and the fact that the younger public, though digital, also report on not finding relevant cultural offers are of high significance. Social media open windows but also build barriers to reaching new audiences and engaging those who are less advantaged. Therefore, a good deal of creativity and informed choices should define the communication strategies of CCIs in the future. 
The study confirms that optional digital access to cultural events held in situ is likely to become a new norm. While preferences towards events in situ are abundant, the new status quo has already shaped audience expectations that those who cannot attend physically should be able to access the content /event digitally. Thus, the online public is here to stay.

Cultural operators will have to consider ways to make cultural attendance and participation possible for more and diverse types of creative works and events. While this is challenging, it also provides an opportunity for diversifying cultural offers. There are new streams of revenue too as the online public is considerably larger, if not unlimited. As cultural goods and services evolve, the CCI organizations and creative professionals would need to keep abreast with the audience's expectation to stay informed (online and offline) of new cultural offers meeting their interests. This means investing in activities that activate the public and cultivate it in new ways.

The key barrier to cultural attendance is the technical quality of online offers. Respondents framed this to be the new factor distancing them from the cultural experience. By contrast, the risk of offering creative experimentation online is likely to present less of an obstacle for public activation compared to the in-situ engagement with culture. Audiences are more willing to invest attention and time in experimental artworks or new cultural offers online as this participation requires a lower financial commitment and provides the public with a certain anonymity. CCI organizations may benefit from this trend and direct certain offers (first or only) to the online public to test initial interest or benefit from the larger audience.

The study validates a trend for demand of additional offers to accompany the core cultural content. There is an expressed public interest in in-depth materials such as lectures and discussions around the artwork and the creative processes. Cultivating this public demand by delivering educational content online is a direct call to organizations to engage with providing behind-the-scenes and process-based content interactive formats. In addition, innovation projects will stimulate the overall renewal of the economy as they are best realized in joint or network entrepreneurship which stabilizes the CCI's organizational development (Koleva, 2013).

A key conclusion of the study is that public attendance - now either fully or partially online - induces structural changes in the CCI value chain and work processes. One should note that across the developed world a specific "problem of public support schemes" has been flagged. They are seen as 'ill-adapted to CCS business models and forms of employment and in need of reform. (OECD, 2020) Specifically, innovation support for new creative formats and content, including the use of different media, new ways of working, new business models, and new forms of co-production are considered of high priority in the current context of policy decisions and support.

Among the specific attention points is the emerging interest in cultural offers that capture the attention but avoid accruing digital fatigue. To meet this and the abovestated expectations of the public and step into new trends. CCIs need new resources and tools. They are already acquiring new skills and seeking funds and support to enhance their capabilities for delivering enhanced cultural offers online. New forms of the offer, such as in series or episodes, are likely to transform public participation. They, of course, require serious investment from CCIs which most of them are not able to make on an individual basis.

Clear recommendations have been flagged again for "public sector initiatives to support these processes and create new platforms for dissemination of creative content ... [prioritizing] digital infrastructure" (OECD 2020). Given the local context in Bulgaria, it is noteworthy that this very same priority was formulated by the large collective representing the free arts scene of Sofia back in 2016. Today this strategy has been endorsed by the municipality. Yet, the target investments in promoting all CCI 
organizations and their offer via a shared communication platform connecting them to audiences in Sofia and attracting new public interest in its arts scene are still being negotiated (Koleva et al., 2016). Public support is essential to the CCIs as it is apparent that the quality of local offers enters competition across geographical or linguistic boundaries. This means that without specific action, the local public may become estranged and disadvantaged while local CCIs would either become more innovative or close operations.

The individual sections of the research analysis presented in this article focused only on the key results regarding public preferences for attending cultural events online and/or use of cultural content online; the motivations and barriers to culture access online and finally new formats of interest to the audience. In the course of the analysis, specific indications were provided on emerging trends and demands that define the newly shaped dispositions of the public, including a comparison of the view of the same public on what they value and appreciate the most from cultural participation online / in situ. The authors expect that this survey has captured an imprint of the 'public dispositions' at a very crucial moment when the audience still recalls the period of time before online cultural participation became a norm.

\section{References}

Auxier, B., \& Silverglate, P., (2021, August). About one-third of consumers report feeling overwhelmed by tech management during COVID-19, Deloitte. https://www2.deloitte.com/xe/en/insights/industry/technology/digitalfatigue.html

Berbenkova, M., \& Koleva, P. (2020, April). Отново свързани: културният сектор в извънредно положение [Reconnect: the cultural sector in a state of emergency]. https://inter-cultura.eu/reconnect-the-cultural-sector-in-a-stateof-emergency/

Bourdieu, P. (1987). Distinction: A social critique of the judgment of taste. Harvard University Press

Brookes, A. (2008). The Public Values of Controversial Arts. In M. Hutter \& D. Throsby (Eds.), Beyond Price: Value in Culture, Economics, and the Arts (ch. 16, pp. 270 282), Cambridge University Press. https://doi.org/10.1017/CB09780511793820.017

European Commission. (2010). Unlocking the potential of cultural and creative industries, European Commission DG EAC. Green Paper COM. https://op.europa.eu/en/publication-detail/-/publication/1cb6f484-074b4913-87b3-344ccf020eef/language-en

IDEA Consult, Amann, S., \& Heinsius, J. (2021). Creative FLIP Final Report, Work Package 1 on Finance, Brussels. http://creativeflip.creativehubs.net/wpcontent/uploads/2021/07/BUILD_BETTER_WITH_INNOVATION.pdf

Intercultura Consult. (2020). Reconnect: Audiences and Cultural Content in the Digital Environment. https://inter-cultura.eu/reconnect/

Koleva, P. (2013). Иновационни проекти като фактор за стратегическо развитие на организации в културния сектор [Innovation projects as a strategic development factor for cultural organisations], Orgon/Intercultura Consult, $01 / 2013$.

Koleva, P., et al. (2016). Споделена визия стратегия за развитие на свободната творческа сцена [Shared Vision - Municipal strategy developing the independent arts scene of Sofia]. https://www.sofia.bg/documents/20182/890150/1cSV Book Web.pdf/63a2e322-fe28-4681-a6bb-8aff47d9eff6

Koleva, P., Sandell, T., Bardun, Y., Di Nola, M., \& Gaeta, J. (2021, March). Creative and cultural industries cross-innovations. Collaboration for sustainable growth in the Northern Dimension countries. Arts Management Quarterly, 136: Serving Communities, 4317. https://www.artsmanagement.net/Articles/Creative-and- 
cultural-industries-cross-innovations-Collaboration-for-sustainable-growth-inthe-Northern-Dimension-countries

Lhermitte, M., Alvarez, J., Marcout, C., Nam, Q., \& Sauze, E. (2021, January). Rebuilding Europe. The cultural and creative economy before and after the COVID-19 crisis. https://1761b814-bfb6-43fc-9f9a775d1abca7ab.filesusr.com/ugd/4b2ba2 1ca8a0803d8b4ced9d2b683db60c18 ae.pdf

Lorenzen, M., \& Friederiksen, L. (2007). Why do Cultural Industries Cluster? Localization, Urbanization, Products, and Projects. In P. Cooke, \& L. Lazzeretti (Eds.), Creative Cities, Cultural Clusters and Local Economic Development (pp. 155 - 177), Edward Elgar.

McAndrew, C. (2020). The Impact of COVID-19 on the Gallery Sector A 2020 mid-year survey. Art Basel and UBS. https://d2u3kfwd92fzu7.cloudfront.net/The Art Market Mid Year Survey 202 0-1.pdf

McSpadden, K. (2015, May 14). You Now Have a Shorter Attention Span Than a Goldfish, Time Magazine. https://time.com/3858309/attention-spans-goldfish/

OECD. (2020, September 7). Culture shock: COVID-19 and the cultural and creative sectors. https://www.oecd.org/coronavirus/policy-responses/culture-shockcovid-19-and-the-cultural-and-creative-sectors-08da9e0e/

PRODISS. (2020, March 20). COVID-19 590 millions d'euros de pertes de chiffre d'affaires estimées pour le secteur du spectacle vivant privé, et 37900 personnes concernées par un arrêt de leur activité [COVID-19 $€ 590$ million in estimated loss of turnover for the private performing arts sector, and 37,900 people affected by a cessation of their activity]. http://www.prodiss.org/sites/default/files/atoms/files/cp - prodiss 200320 - spectacle vivant prive 590 meu de pertes de ca.pdf

Sofaer, J., Davenport, B., Sørensen, M., Gallou, E., \& Uzzell, D. (2021). Heritage sites, value and wellbeing: learning from the COVID-19 pandemic in England. International Journal of Heritage Studies, 27(11), 1117-1132. https://doi.org/10.1080/13527258.2021.1955729

Stoeva, N. (2020, May). Analysis of the survey of the Independent Sector for the period 22.03.2020 - 17.04.2020, https://bit.ly/3cohTCI

Wiesand, A., \& Ateca-Amestoy, V. (2021). HERIWELL Cultural Heritage as a Source of Societal Well-being in European Regions. Survey to population in 8 ESPON countries, presented in the workshop on October 4, 2021. www.espon.eu/HERIWELL

\section{Acknowledgments}

This article contains data and analytical summary from the survey executed by: Milena Berbenkova, Petya Koleva, Nelly Stoeva, Petya Boyukova, Lidiya Peycheva, Vesela Deyanova, and Diana Nedeva, with support from Emil Denev. The Creative partners in the project were Contemporary Alternative Art and Culture Organization "36 Monkeys", the puppet theatre "MalleMalle", and Meeting Points foundation. These results were part of "Reconnect: Audiences and Cultural Content in the Digital Environment", a project developed and led by Intercultura Consult was financed by the National "Culture" Fund, Bulgaria. https://inter-cultura.eu/reconnect/. Reconnect: Audiences and Cultural Content in the Digital Environment https://intercultura.eu/reconnect/

\section{Author biographies}

Petya Koleva, Ph.D., is an international expert based in Sofia, over the last 18 years nurturing international research and collaboration, engaging with arts organizations and with cultural policymakers. She is the founder of Intercultura Consult (2004), specializing in research and 
consultancy, developing synergies between arts and innovation. At the EU level, Dr. Petya Koleva has recently been involved with EU funded NDPC project "Cultural and Creative Industries (CCI) Cooperation and Innovation in the Northern Dimension Countries", the i-Portunus, program for the mobility of arts and culture professionals of Creative Europe and the project valuating Cultural Heritage as a Source of Societal Well-being in European Regions - ESPON HERIWELL.

Milena Berbenkova is an audience analysis and development specialist. She coordinated the project "Reconnect: Audiences and Cultural Content in the Digital Environment" and is now the manager of its successor "Time Perspectives: Long-term Benefits of the Culture - Audience Relationship". She has international experience in working with CCIs in the performing arts, tourism, and museum sector.

(C) 2021 Faculty of Management (SNSPA), Author(s). This is an open-access article licensed under the Creative Commons Attribution-NonCommercial-NoDerivs License (http://creativecommons.org/licenses/by-nc$\underline{\text { nd } / 4.0 /)}$. 